\title{
Revisiting Constraints of Fourth-Generation Quarks and Leptons
}

\author{
Aayush Verma \\ India
}

Email: aayushverma6380@gmail.com

\begin{abstract}
Standard Model is well-performing under the three-generation, but in the wake of more physics, fourth-generation leptons and quarks are introduced. In this paper, I created a model surrounding quarks and leptons and tried to show the importance of quarks to understand the baryogenesis. I talked about and set the lower limits of t' and b'. And predicted an updated version of lower bounds and precise masses of quarks and leptons. In Sec. II. I mathematically predicted the Higgs boson anomaly in the new standard model due to neutrinos and leptons. And in final, I talked about the Unified Standard Model.
\end{abstract}

Keyword Beyond the Standard Model; Fourth Generation Particles; Lepton Sector; Quarks Sector

\section{INTRODUCTION}

In recent years the SM4, the upgraded version of SM3 has been in point of attraction by many physicists. After the last piece, Higgs Boson in the SM3, there are many questions in paucity about the masses of next Leptons and Quarks. In SM3, the mass of Higgs Boson is $125.18 \pm 0.16 \mathrm{GeV}$ [1], but there is quite an update that we will encounter with the Higgs masses. In SM4, the mass of Higgs Boson is around $115 \mathrm{GeV}$ (we will examine it later). With the extension of SM3 to SM4, we just add a couple of Leptons and Quarks, Quarks t' and b' with isopin of $\frac{1}{2}$ and $-\frac{1}{2}$ respectively and Lepton l' and its corresponding Neutrino. There are a lot of discussions about the masses and flavor mixing of leptons and quarks. In our model, we will be looking at the current Koide Formula (Hypothesis) 2]:

$$
Q=\frac{m_{e}+m_{\mu}+m_{\tau}}{\left(\sqrt{m_{e}}+\sqrt{m_{\mu}}+\sqrt{m_{\tau}}\right)^{2}} \approx 0.666661 \approx \frac{2}{3}
$$

for theoretical masses of leptons and quarks, albeit the validity of Koide Hypothesis is not yet determined, but this is the incredible yet not definable formula. If Koide formula holds true then calculated mass of $\mathrm{b}^{\prime} \approx 3.6 \mathrm{TeV}$ and mass of $\mathrm{t}^{\prime} \approx 84 \mathrm{TeV}$, perhaps it is common to take lower bound of b' to be $>1.3-1.6$ $\mathrm{TeV}$ (precisely lower bound of b' excluded at 338-362 $\mathrm{GeV}$, see more in reference [3]). The leptonic mass for l' and Neutrino $V_{l}$ ' is quite an in danger because of LHC constraints, LEP experiment using the width of $\mathrm{Z}$ Boson has measured that total number of Neutrino must be 3 [4], with high precision.

But there is a way to cancel the anomaly, we can assume that the mixing of this neutrino is so less with another neutrino, so we have to assume that its mass must be big enough. Also charged lepton, $m_{l^{\prime}}<100$ $\mathrm{GeV}$ is also excluded by another LEP experiment. Hence we must put the lower bound of lepton and neutrino $m_{v_{l^{\prime}}} \leq \frac{1}{2} M_{Z}$. We can assume the identical lower bound for charged lepton $m_{l^{\prime}} \sim \frac{1}{2} M_{Z}$. But there is another anomaly, if the mass is correct, then
Higgs Boson would decay to a pair of neutrinos.

The reason for taking these masses of quarks a thousand times the third generation quarks is because we want to know the Baryon Symmetry CP Violation, which is a strong candidate for Sakharov conditions [5]. The CP Violation is suppressed by s, c, and b quarks because of their low masses [6]. If these new quarks are proven(which is not possible now) then we can admit that baryon asymmetry is the reason because of these heavy quarks. It is widely accepted that CP Violation is necessary to get the Baryogenesis.

Although it has been challenged that $N_{v}=3$, many physicists including me still rely on that neutrinos are not yet studied well in the frame of SM3. Also, the concept of $10^{13}$ or more gain in CPV strictly made us favor SM4 over SM3. If we ignore few parameters and anomalies(that we can't) then, SM4 is perfectly good for understanding the early universe as well as digging more literature in Physics.

There are many models like Axion, X17 and etc. But this one is extremely intresting, so there is a need of current research and development of these particles. We hope to find the results of existence of fourth generation Quarks and Leptons by LHC by 2025 .

\section{HIGGS BOSON IN SM4}

The anomaly we discussed above contains a whole set of new literature. That eventually rule out whole leptons discussion. In SM4, our assumed $M_{H} \approx 115$ $\mathrm{GeV}$ and the decay branch $H \rightarrow v^{\prime} \bar{v}^{\prime}$. Along with $H \rightarrow b \bar{b}, H \rightarrow g g, H \rightarrow \gamma \gamma, H \rightarrow v^{\prime} \bar{v}^{\prime}, H \rightarrow W W$ and $H \rightarrow Z Z$, Higgs Boson gets more complicated as a scalar (you can check the branching ratios in Fig 1). In FIG. 1. the masses are updated with SM4 parameters, where heavy leptons masses are set to $m_{l^{\prime}}=m_{v^{\prime}}+$ $50 \mathrm{GeV}$ [4]. Although the fate of the $H \rightarrow v^{\prime} \bar{v}^{\prime}$ is criticized a lot with the data of ATLAS and CMS, this branching can introduce a lot of new chain reaction as this heavy neutrino get further decay(but in this 
case of fourth-generation $v_{l^{\prime}}^{\prime}$ it might not). Talking about this neutrino is so dangerous, as if the mass of the neutrino is too large then it will suppress by the factor $<10$ to decay. If the new heavy neutrino doesn't decay then weak interactions of other decays of neutrinos $\left(v_{e}, v_{\mu}, v_{\tau}\right)$ becomes a mystery, it is quite not a possible

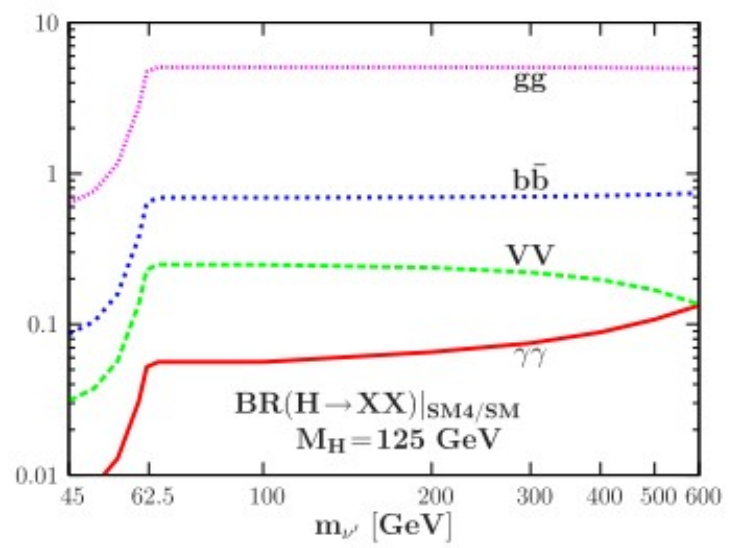

FIG. 1. Higgs Boson decay. branching ratio into gg, $b \bar{b}$, $\mathrm{VV}$, where $\mathrm{V}=\mathrm{W}, \mathrm{X}$.

thought that some neutrino does decay others don't. Hence it is quite tough to explain the fourth generation leptons, so we simply exclude these leptons because ATLAS and CMS reported (see ref [7], 8]) $H \rightarrow \gamma \gamma$ signal with a significance of more than 4 standard deviations from the SM4. The factor observed was $1.9 \pm 0.5$ for ATLAS and $1.56 \pm 0.43$ for CMS which is much low in SM4, maybe because of channel $H \rightarrow v^{\prime} \overline{v^{\prime}}$ which is not appropriate for Standard Model. Also in SM4, one must expect more enhanced data of $H \rightarrow \tau \tau$ channel which is not observed in current data. So our assumption of $m_{v^{\prime}} \approx \frac{1}{2} M_{z}$ is kind of false, see reference for more [4]. This alone seals the fate of fourth-generation leptons, along with the accurate prediction of $N_{v}=3[9]$ by $\mathrm{Z}$ width(LEP). But I still doubt the insufficient experimental data and quite of favor SM4 in the wake of more study of $v_{l}^{\prime}$ neutrino, the matter of the existence of this neutrino depends on the mass of this neutrino. But for now, fourth-generation leptons are not quite likely to be found in accelerators.

There is also another possibility of being it from another model, not from SM4, actually this neutrino would have negligible coupling to any observed particle of SM3. Or it have it's own coupling family, I mean to say an another particle physics model, which we are not ready for.

\section{QUARKS IN SM4}

While you see the lepton fourth-generation section is destroyed with observational data, there are still possibilities of finding heavy quarks of masses that we have assumed. Quarks are the way and in my way, it is the only way to understand the dominance of matter over anti-matter, i.e Baryogenesis [10. If this holds true then, our 6 flavors quarks with gluons of $\mathrm{SU}(3)$ symmetry is not yet completed. Well, the question arises, what is the significance of heavy quarks and why we need them?

The heaviest quark we have is a top quark with a mass of $173.2 \pm 1.0 \mathrm{GeV}$ with its ad-joint quark with a second-heaviest bottom quark with a mass of $4.20 \pm 0.04 \mathrm{GeV}$. These two quarks are not much found, but does make us understand the strong force and other phenomena.

Using the view of an experimentalist, it is quite easy to tell that our standard model is complete. But theoretically, it is not yet ready, as the current standard model not precisely tell us the answer related to questions like:

1. Exclusion of gravity.

2. Why there is not any specified coupling constant for gravity in field theory?

\section{Dominance of matter over anti-matter}

4. Maybe why and how dark matter behave?

The gravity thing and the coupling constant concept are the goals of now-days physicists. It is called the unification of gravity with other forces. But no theories such as supersymmetry, string theory (although it is the best candidate for unification) can prove GUT, perhaps not now. So our clear indication goes to Standard Model (albeit all those candidate presequites is particle physics, but many physicists goal to have unification on the solely base of particle physics) for unification, more concretely using the particles and quarks system that we have and that we haven't.

I will more emphasize here on the 3rd list. That is the dominance of matter over anti-matter. In SM3 the heaviest mass is $173.2 \mathrm{GeV}$, and Sakharov's condition [5] of CP Violation doesn't favor this mass. I can cite many papers and articles [6], [11] where the heaviest quarks are needed to properly guess the baryon to anti-baryon ratio. If we prefer b' and t', then CP Violation must have some key role in the early universe.

It can be seen that for every baryon there are $2 \times 10^{9}$ photons at 2.7 K in CMBR (Cosmic Microwave Background Radiation) [12,

$$
\frac{n_{B}}{n_{\gamma}}=5 \pm 0.3 \times 10^{-10}
$$

however, we don't see that much anti-baryon in this scenario. However, we have some sufficient things in our current SM model, which can define this anomaly. But to a certain level, and we see that s, b and $\mathrm{c}$ are suppressed by a factor of $10^{-13}$ (if I am not wrong) 
because of their masses $95_{-3}^{+9} \mathrm{GeV}, 4.18_{-0.03}^{+0.04} \mathrm{GeV}$ and $1.275_{-0.035}^{+0.025} \mathrm{GeV}$ respectively. Believe me, it is all about the masses that come into the play, if we just compare strange mass to lower bound of b' with $\mathrm{s}$ quark, then the ratio comes,

$$
\frac{m_{b^{\prime}}^{l b}}{m_{s}} \geq 15.3
$$

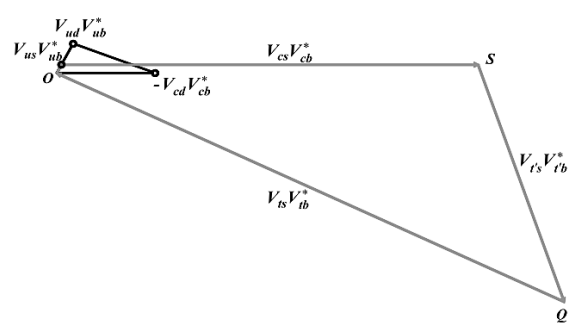

FIG. 2. A Figure representing the knowledge of our confined to the triangle, compared out the fourth-generation model in context of CPV.

and if we take our hypothetical mass then it goes to almost 35 orders. Where $m^{l b}$ is the lower bound mass of b'. Similarly in the case of t', it is perhaps even bigger or biggest ratio, i.e the order of 854 . The ratio of ' ' and t quark is somewhere 482 with \pm 10 . You can judge how the big mass would be. This is just speculative and pretty awesome.

At this point, we can't talk about the decays mode of this b' and t' but in pp collision at center of mass

of $7 \mathrm{TeV}$, one can predict $b^{\prime} \bar{b}^{\prime} \rightarrow t W^{-} t W^{+}$which in further decays to $b W^{+} W^{-} \bar{b} W^{+} W^{-}[3]$. We can't possibly (I mean factfully) talk about decays of t' unless and until we have precise upper bound and lower bound of t'. But we can say that decay would have $t$ quark and boson (simply $\mathrm{W}$ or $\mathrm{Z}$ ). But one possibility is determined, if $m_{t}^{\prime}>m_{b}$, , then $t^{\prime} \rightarrow b^{\prime} W$. And there is a particularly neat decay of b' when the mass splitting is bigger than $m_{W}$, then $b^{\prime} \rightarrow t^{\prime} W^{-}$and the quite twin result we have seen above. Another possibility is when $m_{b^{\prime}}+m_{W}>m_{t^{\prime}}>m_{b^{\prime}}$, then the decay would have $t^{\prime} \rightarrow q W^{+}$, where $\mathrm{q}=\mathrm{c}, \mathrm{t}, \mathrm{u}$ [13. Other possibilities are:

$$
\begin{aligned}
& \text { - } t^{\prime} \rightarrow b^{\prime} W^{*} \text { where } W^{*} \text { is a virtual W-Boson. } \\
& \text { - } t^{\prime} \rightarrow q W^{+} \text {where } \mathrm{q}=\mathrm{d}, \mathrm{s}, \mathrm{b} .
\end{aligned}
$$

But in our model, the first ones are considered to be of high precision and mostly found. Perhaps, if we question the heavy mass of $\mathrm{t}$ quark, $\mathrm{W}$ and $\mathrm{Z}$ boson, then we can say that they are the product of the heaviest quark t' (in this scenario).

Back to the Sakharov Conditions, CP invariance, according to the idea, the universe, more precisely the hot expanding universe* was not invariant under CP symmetry. Although we have not any experimental evidence for this (besides some legit observations), various decays are reported claiming this.

In case of our CPV which fell sort of $10^{-10}$ after the best effort in SM3. By including two more CPV phases as compared to SM3, Baryogenesis is possible with the dynamics that already exist in the Standard Model (current one). So we say that including the fourth generation is interesting and a small revolution as a whole. Learning about the electroweak symmetry and baryon asymmetry is the key to the standard model. Fourth-generation quarks are even interesting, as one gets a $\mathrm{CPV}$ factor gain of $10^{10}$ or even more. Indeed there is a very interesting figure representing the knowledge of ours as compared to the fourth generation CPV knowledge, see FIG. 2.

\section{THE UNIFIED STANDARD MODEL}

After discussing our constraints and putting the number of ideas with the available and non-available option, it can be seen that the physics of particles are not complete. Our current model scales from $0.1 \mathrm{MeV}$ to $173 \mathrm{GeV}$ and the mass difference is about 83827 $\mathrm{GeV}$ from t to the next generation t'. It can be noted that my primary focus is mass. Because the difference is amazing and if the LHC in the future can predict these quarks, it is then going to be a completely new generation for new generation physicists. But one common question arises.

\section{Is Fourth Generation Quarks possible without Fourth Generation Leptons?}

As we have revisited the whole problem with my perspective, and I still believe in fourth-generation leptons (theoretically). But I have myself declared that more mathematics is needed to build the foundation for heavy leptons such mass as $\frac{1}{2} M_{z}$ and $\frac{1}{2} M_{H}$. The parameters are not that free. So we excluded it. But there is a history revolving around, there are equal generations of quarks and leptons. Can we violate it?

I think of course, I certainly don't see that as a mathematical boundary, so no one is obliged to not cross that statement. However, if we take an additional gauge group $\mathrm{SU}(5)$ in the model, things get better. Like we get the mass that we want and we get the possibility of freedom in fourth-generation quarks [14] (But things get more complicated as SU(5) breaks the symmetry, more in Appendix 1).

In short, it is possible. But only theoretically now, but soon it may turn out to be wrong or just a fantastic right. And the Unified Standard Model, then would have at least 14-16 particles. And we may get closer to asymptotically freely QCD. It may be a dream of a theorist to be considered correct in this case ( $\mathrm{I}$ am from this group) and a determination of experimental- 
ist at the accelerator. Albeit LHC or any accelerators are not enough to produce these heavy masses, so we have wait until LHC operates at this masses.

\section{CONCLUSION}

We started with masses of various fourth-generation quarks and leptons, by mixing it with our idea of mass. And found the lower bound to be $1.3 \mathrm{TeV}$ for b' and 4 TeV for t' (in our model, however, we discussed other models as well). Then we examnined the mass of neutrino and leptons of fourth-generation and constrained it at the mass of half the Z-Boson. After that, we performed the Higgs Boson scenario in SM4 with its updated mass $115 \mathrm{GeV}$ and showed the anomaly. Then we dropped the idea of fourth-generation quarks. We talked about the existing constrained value and tried to explore it. Later we talked about the new physics that can be unlocked by including fourth-generation quarks and proved how successful it will be. Then we measured the various parameters ratio and found it much bigger than any ratio we have seen in the con- text of particles. And In the end, we talked about the possibility of quarks without leptons and predicted t' and b' to be interesting and if found then it will be another victory of physics.

\section{APPENDIX 1}

Flavor Symmetry is one of the biggest problem in QCD. While $\mathrm{SU}(2)$ seems to be good, SU(3), SU(4) and so on terribly break the symmetry in particle physics. Especially in Quarks Section, SU(4) and so on gives us a terrible break in symmetry. The conditions and the masses we are reviewing here, also don't follow any symmetry, as $\mathrm{SU}(8)$ is very far complicated to be fitted in Symmetry even though we haven't even succeeded in the SU(4). The masses difference between quarks after down quarks are very large, and in SM4 it gets more difference. We have at least 80 orders of difference between t' and b'. So there is not any chance of flavor symmetry in SM4 either. So the isospin of these quarks are also $0, I=0$.
[1] M. Aaboud, G. Aad, B. Abbott, O. Abdinov, B. Abeloos, S. H. Abidi, O. AbouZeid, N. L. Abraham, H. Abramowicz, H. Abreu, et al., Physics Letters B 784, 345 (2018).

[2] E. Ma, Physics Letters, Section B: Nuclear, Elementary Particle and High-Energy Physics 649, 287 (2007)

[3] Search for a Heavy Bottom-like Quark in pp Collisions at $\operatorname{sqrt}(s)=7 \mathrm{TeV}$, Tech. Rep. CMS-PAS-EXO-11036 (CERN, Geneva, 2011).

[4] A. Djouadi and A. Lenz, Physics Letters B 715, 310 (2012).

[5] A. D. Sakharov, Journal of Experimental and Theoretical Physics Letters (JETP Letters) , 24 (1967).

[6] G. W. Hou, International Journal of Modern Physics D 20, 1521 (2011).

[7] V. A. Khoze, arXiv preprint hep-ph/0105069 (2001).

[8] G. Guo, B. Ren, and X.-G. He, arXiv preprint arXiv:1112.3188 (2011).
[9] K. Nakamura, K. Hagiwara, K. Hikasa, H. Murayama, M. Tanabashi, T. Watari, C. Amsler, M. Antonelli, D. Asner, H. Baer, et al., Journal of Physics G: Nuclear and Particle Physics 37, 075021 (2010).

[10] V. M. Abazov, B. Abbott, M. Abolins, B. S. Acharya, M. Adams, T. Adams, E. Aguilo, G. Alexeev, G. Alkhazov, A. Alton, et al., Physical Review D 82, 032001 (2010).

[11] S. Pandolfi, New source of asymmetry between matter and antimatter, https: //home.cern/news/news/experiments/ new-source-asymmetry-between-matter-and-antimatter (2017).

[12] C. Bennett, M. Halpern, G. Hinshaw, N. Jarosik, A. Kogut, M. Limon, S. Meyer, L. Page, D. Spergel, G. Tucker, et al., arXiv preprint astro-ph/0302207 (2003).

[13] D. Atwood, S. K. Gupta, and A. Soni, Journal of High Energy Physics 2012, 105 (2012).

[14] C. D. Froggatt, H. Nielsen, and D. Smith, Zeitschrift für Physik C Particles and Fields 73, 333 (1997). 